\title{
Activation of the Nrf2-ARE Pathway Attenuates Hyperglycemia-Mediated Injuries in Mouse Podocytes
}

\author{
Cheng Wanga CuiCui Lia, Hui Peng ${ }^{a}$ Zengchun Yea Jun Zhanga Xun Liua \\ Tanqi Lou ${ }^{\mathrm{a}}$ \\ aDivision of Nephrology, Department of Medicine, the $3^{\text {rd }}$ Affiliated Hospital of Sun Yat-Sen University, \\ Guangzhou, Guangdong, China, ${ }^{b}$ Division of Nephrology, Department of Medicine, the $5^{\text {th }}$ Affiliated \\ Hospital of Guangzhou Medical University, Guangzhou, Guangdong, China
}

\section{Key Words}

Antioxidant response element - High glucose - Nuclear factor (erythroid-derived 2)-like 2 • Podocyte $\cdot$ Tert-Butylhydroquinone

\begin{abstract}
Background: Damage to podocytes caused by excessive reactive oxygen species (ROS) contributes to onset and progression of diabetic kidney disease (DKD). Nuclear factor (erythroid-derived 2)-like 2 (Nrf2) is a redox-sensing transcription factor that can induce the expression of antioxidant enzymes. We explored whether activation of Nrf2 pathway attenuated hyperglycemia-induced injuries in mouse podocytes. Methods: Tert-Butylhydroquinone (tBHQ) and small interfering RNAs (siRNAs) were used to regulate Nrf2 expression. Apoptosis and intracellular superoxide anion production were measured by flow cytometry. The activity of the Nrf2 antioxidant pathway was measured by an antioxidant response element (ARE)driven luciferase reporter gene assay, and Nrf2 expression was assessed by real-time PCR and western blot analyses. Results: Podocytes incubated with high-glucose (HG) medium had higher intracellular superoxide anion and hydrogen peroxide production, higher apoptosis rate, higher bovine serum albumin (BSA) permeability and lower synaptopodin expression compared with podocytes exposed normal glucose $(N G)(p<0.05)$. tBHQ increased the activity of the Nrf2 antioxidant pathway and enhanced nuclear Nrf2 expression, reduced intracellular superoxide anion and hydrogen peroxide production, apoptosis rate and BSA permeability, and restored synaptopodin expression in podocytes exposed to HG $(p<0.05)$. Podocytes with Nrf2 siRNAs showed higher intracellular superoxide anion and hydrogen peroxide production, apoptosis and BSA permeability as well as lower synaptopodin expression compared with podocytes exposed to HG $(p<0.05)$. Conclusions: Our findings suggest that protection against activation of the Nrf2-ARE pathway in podocytes exposed to hyperglycemia. Thus, regulation of of the Nrf2-ARE pathway could be a therapeutic option to combat oxidative stress and inhibit the development of DKD.
\end{abstract}

C. Wang and C. Li contributed equally to the manuscript. 


\section{Cellular Physiology and Biochemistry}

Cell Physiol Biochem 2014;34:891-902

\begin{tabular}{l|l}
\hline DOI: $10.1159 / 000366307$ & C 2014 S. Karger AG, Basel
\end{tabular}

www.karger.com/cpb

\section{Introduction}

The role of hyperglycemia in long-term complications has been established by largescale prospective studies for type-1 and type-2 diabetes mellitus (DM) [1]. Hyperglycemia engenders not only excessive free-radical factors but also attenuates anti-oxidative factors [2]. Hence, oxidative damage from free-radical and non-radical oxygen species contributes to the pathogenesis of diabetic complications, including the onset and progression of diabetic kidney disease (DKD) [3]. Experimental and clinical studies have documented a link between hyperglycemia, oxidative stress and DKD [4].

Podocytes are terminally differentiated and play a key part in preserving glomerular capillary integrity as well as formation of the glomerular filtration barrier [5]. Injury to podocytes is considered a major contributor to the initiation and progression of DKD [6]. In various experimental animal models of DM, podocyte loss is followed by the remaining podocytes trying to cover a larger surface area of the glomerular basement membrane. Subsequently, the podocyte "foot" widens, which is followed by proteinuria and progressive glomerulosclerosis [7]. Early glomerular changes in animal models of DM are characterized by podocyte injury without mesangial expansion [8]. Patients with early type- 1 and -2 DM experience a loss of podocyte number. A correlation between the rate of albumin excretion and the drop in podocyte number has been demonstrated [9]. Taken together, these data indicate that podocyte injury has a role in the onset and progression of DKD. Nuclear factor (erythroid-derived 2)-like 2 (Nrf2) is a membrane of the cap ' $n$ ' collar type of basic region leucine zipper factor family. Nrf2 is expressed broadly in tissue but is activated only in response to a range of oxidative stimuli [10]. Upon activation, Nrf2 translocates to the nucleus and binds to the antioxidant response element (ARE), which mediates the induction of a spectrum of cytoprotective proteins: the reduced form of nicotinamide adenine dinucleotide phosphate (NADPH):quinone oxidoreductase, heme oxygenase [11], glutathione reductase, superoxide dismutase (SOD) and catalase (CAT) [12]. Impairment of the Nrf2-ARE pathway has been observed in retinal endothelial cells, H9c2 cells, mesangial cells and coronary arterial endothelial cells induced by hyperglycemia, and glucose-induced impairments are prevented by activation of the Nrf2 pathway [13-16]. However, the role of activation of the Nrf2-ARE pathway in podocyte injury induced by hyperglycemia is not known. Hence, we hypothesized that activation of the Nrf2-ARE pathway in podocytes can alleviate damages induced by high glucose.

\section{Materials and Methods}

\section{Chemicals and reagents}

Tert-Butylhydroquinone (tBHQ) and N-acetylcysteine (NAC) were purchased from Sigma-Aldrich (St. Louis, MO, USA). Dihydroethidium (DHE) was used as sensitive proble for superoxide anion (Molecular Probes Inc, Eugene, OR). The Amplex® Red Hydrogen Peroxide/Peroxidase Assay Kit was from Life Technologies (Life Tech, CA). Fluorescein isothiocyanate-bovine serum albumin (FITC-BSA) was obtained from Nordic Immunology (Tilburg, the Netherlands). Commercially available malondialdehyde (MDA), SOD, glutathione (GSH) and CAT kits were purchased from Nanjing Keygen Biotech (Nanjing, China), as were lysis buffer and nucleoprotein extraction kits.

Primary polyclonal rabbit antibodies for Nrf2, synaptopodin and lactate dehydrogenase (LDH) were obtained from Santa Cruz Biotechnology (Santa Cruz, CA, USA). Rabbit anti-heme oxygenase-1 (HO-1) and anti-lamin B1 antibodies were obtained from Cell Signaling Technology (Beverley, MA, USA). Rabbit antiNADPH:quinine oxidoreductase (NQO1) antibody was purchased from Abcam (Cambridge, MA, USA) and anti-glyceraldehyde 3-phosphate dehydrogenase (GAPDH) antibody was from Proteintech (Chicago, IL, USA). Horseradish peroxidase-conjugated secondary antibody (goat anti-rabbit) and enhanced chemiluminescent reagent were purchased from Pierce (New York, NY, USA). An annexin-FITC Apoptosis Detection kit was obtained from BD Biosciences (San Jose, CA, USA). The specific Nrf2 small-interfering RNA (siRNA) negative control of siRNA, Lipofectamin ${ }^{\mathrm{TM}}$ RNAiMAX Reagent, and Lipofectamine 2000 Reagent were purchased 


\section{Cellular Physiology and Biochemistry}

Cell Physiol Biochem 2014;34:891-902

\begin{tabular}{l|l}
\hline DOI: $10.1159 / 000366307$ & (c) 2014 S. Karger AG, Basel
\end{tabular}

www.karger.com/cpb

Wang et al.: Nrf2 in Podocytes with Glucose

from Invitrogen (Carlsbad, CA, USA). An ARE Reporter Kit was purchased from BPS Bioscience (San Diego, CA, USA). A Dual-Glo® Luciferase Assay system was from Promega (Promega, Madison, WI, USA). Trizol reagent was purchased from Invitrogen. A SYBR Premix Ex Taq kit was obtained from TaKaRa (Shiga, Japan).

\section{Cell cultures}

Mouse podocytes were provided and cultured as described previously [17, 18]. Growth-arrested podocytes were grown in normal glucose (NG; $5 \mathrm{mM}$ ), mannitol (MA; $25 \mathrm{mM}$ ) and high glucose (HG; $25 \mathrm{mM}$ ) medium. The widely used Nrf2 activator tBHQ $(20 \mu \mathrm{M})$ was pretreated for $4 \mathrm{~h}$ to investigate the potential protective role of Nrf2 activation in podocytes mediated by hyperglycemia. The classical antioxidant NAC $(15 \mathrm{mM})$ was used to identify the role of reactive oxygen species (ROS) in HG-mediated podocyte injury. siRNAs were used to downregulate Nrf2 expression in podocytes. All doses and time of these treatments were determined in our pilot studies.

SiRNA transfection

The sequences of Nrf2 siRNA were: sense, $5^{\prime}$-UUGGGAUUCACGCAUAGGAGCA

CUG-3'; antisense, 5'-CAGUGCUCCUAUGCGUGAAUCCCAA-3'. Nrf2 and negative control of siRNA transfection were undertaken using Lipofectamine ${ }^{\mathrm{TM}}$ RNAiMAX Reagent according to manufacturer instructions.

Measurement of intracellular superoxide anion production with DHE

Intracellular superoxide anion production was determined by using the fluorescent probe DHE [19]. Briefly, Cells were collected and then incubated with $10 \mu \mathrm{mol} / \mathrm{L}$ DHE for $20 \mathrm{~min}$ at $37^{\circ} \mathrm{C}$ in the dark. Then, the fluorescence was detected by flow (excitation wavelength $=488 \mathrm{~nm}$; emission wavelength $=610 \mathrm{~nm}$ ). The mean fluorescence intensity was used to quantify the responses by CellQuest software. A minimum of 10000 cells were acquired for each sample.

\section{Measurement of Hydrogen Peroxide $\left(\mathrm{H}_{2} \mathrm{O}_{2}\right)$ with amplex Red}

The Amplex Red assay was determined in a 96 well plate by the reaction of $50 \mu \mathrm{L}$ of Amplex Red working solution with $50 \mu \mathrm{L}$ of assay sample. The reactions were incubated for $30 \mathrm{~min}$ at room temperature, protected from light. After incubation, fluorescence was measured in a fluo-rescence microplate reader using excitation wavelength at $560 \mathrm{~nm}$ and emission detection at $590 \mathrm{~nm}$. A series of diluted H2O2 concentrations was used for a standard curve.

Apoptosis in podocytes as measured by flow cytometry

Apoptosis in podocytes was determined using the Annexin-FITC Apoptosis Detection kit according to the manufacturer's protocol. The combination allowed for differentiation between early apoptotic cells (annexin V-FITC-positive, PI-negative), late apoptotic cells (annexin V- and PI-positive), necrotic cells (annexin V-negative and PI-positive), and viable cells (unstained). The classification of evaluation of podocyte apoptosis was "early" and "advanced" [20].

\section{ARE luciferase reporter gene assays}

The activity of the Nrf2 antioxidant pathway was measured with the ARE Reporter kit according to manufacturer instructions. Briefly, podocytes were plated in 96-well culture plates 1 day before transfection. Cells were then co-transfected with Reporter and Negative Control Reporter using Lipofectamine 2000 Reagent. These transfected cells were then exposed to their respective treatment. Luciferase activities were measured with the Dual-Glo Lucifease Assay system. The luciferase activities of the firefly were normalized to those of the internal control

RNA extraction, CDNA synthesis and real-time polymerase chain reaction (RT-PCR)

Total RNA from cultured podocytes was extracted by Trizol reagent according to the manufacturer's protocol. Total RNA (600 ng) was reverse-transcribed using reverse transcriptase from a SYBR Premix Ex Taq kit. The primers are listed in the following $\left(\left(5^{\prime} \rightarrow 3\right):\right.$ Nrf2, sense: TTTTCCATTCCCGAATTACAGT, antisense: AGGAGATCGATGAGTAAAAATGGT; HO-1, sense: CGTGCTCGAATGAACACTCT antisense: GGAAGCTGAGAGTGAGGACC; NQ01, sense: CAAGTTTGGCCTCTCTGTGG antisense: AAGCTGCGTCTAACT 


\section{Cellular Physiology and Biochemistry}

Cell Physiol Biochem 2014;34:891-902

\begin{tabular}{l|l}
\hline DOI: $10.1159 / 000366307$ & (C) 2014 S. Karger AG, Basel
\end{tabular}

Wang et al.: Nrf2 in Podocytes with Glucose

ATATGT; Synaptopodin, sense: AGAAGCTACAGTTCTGTTCCCGCA antisense: TTCTACAAGAGGCACAAGGCAGGA; GAPDH, sense: TGTGTCCGTCGTGG

ATCTGA antisense: TTGCTGTTGAAGTCGCAGGAG. Quantitative RT-PCR was done using an ABI 7500 Fast Real-Time PCR System (Applied Biosystems) and SYBR Premix Ex Taq. PCR was carried out as reported $[20,21]$

\section{Western blot analyses}

Total cell proteins were extracted from cultured podocytes by the addition of lysis buffer. Cytoplasmic protein and nucleoproteins were extracted using a Nucleoprotein Extraction kit. For western blot analyses, a $10 \%$ sodium dodecyl sulfate-polyacrylamide gel was run under standard conditions by loading $60 \mu \mathrm{g}$ total proteins and $40 \mu \mathrm{g}$ nucleoproteins in each lane. The gel was placed in transfer buffer for $15 \mathrm{~min}$ and transfered to a polyvinylidene fluoride membrane at $100 \mathrm{~V}$ for $1 \mathrm{~h}$. The membrane was rinsed in Tris-buffered saline followed by rinsing in blocking buffer (5\% milk powder) for 5 min. The membrane was immersed in blocking buffer for $1 \mathrm{~h}$ before incubation with primary antibodies overnight at $4^{\circ} \mathrm{C}$. That is: polyclonal rabbit anti-Nrf2 (1:500 dilution); anti-HO-1 (1:1000), anti-NQO1 (1:1000), anti-lamin B1(1:1000), anti-synaptopodin (1:200), anti-LDH (1:500) and anti-GAPDH (1:10000). After rinsing in wash buffer, horseradish peroxidase-conjugated secondary antibody was used for $1 \mathrm{~h}$. After the final wash, the membrane was developed using ECL Reagent, and densitometric analyses conducted using Quantity One software (Bio-Rad, Hercules, CA, USA).

\section{Measurement of cellular levels of MDA, SOD, CAT and GSH}

Commercially available kits were used to measure the cellular levels of MDA, SOD, CAT and GSH in podocytes. Measurements were made according to manufacturer instructions. Data were normalized with the protein concentration of podocyte lysates.

BSA permeability assay

A BSA permeability assay was used to evaluate the filtration barrier function of podocyte monolayers [18]. Briefly, podocytes $\left(5 \times 10^{3}\right)$ were seeded onto collagen-coated trans-well filters (pore size, $3 \mu \mathrm{m}$; Corning, New York, NY, USA) and cultured under differentiation conditions. Cells were washed twice with PBS. Then, the top chamber was refilled with $0.2 \mathrm{~mL}$ of RPMI 1640 medium and the bottom chamber with 1 $\mathrm{ml}$ of RPMI 1640 medium containing $10 \mu \mathrm{g} / \mathrm{mL}$ FITC-BSA, and incubated at $37^{\circ} \mathrm{C}$. After $4 \mathrm{~h}$ of incubation, a small amount of medium from the top chamber was collected and the fluorescence of FITC-BSA determined.

\section{Statistical analyses}

Data are the mean \pm standard error of the mean unless stated otherwise. Statistical differences were assessed using multivariate ANOVA for repeated measures. All $P$-values quoted are two-tailed, and significance defined as $p<0.05$.

\section{Results}

HG induced excessive ROS and damages in mouse podocytes

Podocytes incubated in HG medium had a higher level of intracellular superoxide anion and hydrogen peroxide production compared with podocytes incubated with NG $(p<0.05)$. Simultaneously, HG medium also induced significant damages in these podocytes: higher apoptosis rate, lower synaptopodin expression and higher BSA permeability in these podocytes compared with podocytes exposed to NG $(p<0.05)$ (Fig. 1).

\section{NAC protected mouse podocytes from excessive ROS and damages induced by $H G$}

To identify the role of ROS in podocyte injury, the classical antioxidant reagent NAC were used in these studies. The pretreatment of NAC in podocytes exposed to HG can lower the levels of intracellular superoxide anion and hydrogen peroxide production than in podocytes exposed to $\mathrm{HG}(p<0.05)$, at the same time, pretreatment with NAC reduced 


\section{Cellular Physiology and Biochemistry}

Fig. 1. Comparison of the generation of ROS, apoptosis rate, synaptopodin expression and BSA permeability in podocytes exposed to different treatments. A: Comparison of superoxide anion generation in podocyte from different groups. B: Comparison of hydrogen peroxide production in podocytes from different groups. C: Comparison of apoptosis rate in podocyte from different groups. D: Comparison of synaptopodin mRNA expression in podocyte from different groups. E: Comparison of synaptopodin expression in podocyte from different groups. F: Comparison of BSA permeability in podocyte from different groups. NG: Mouse podocytes incubated with normal glucose ( $5 \mathrm{mM}$ ) for 24
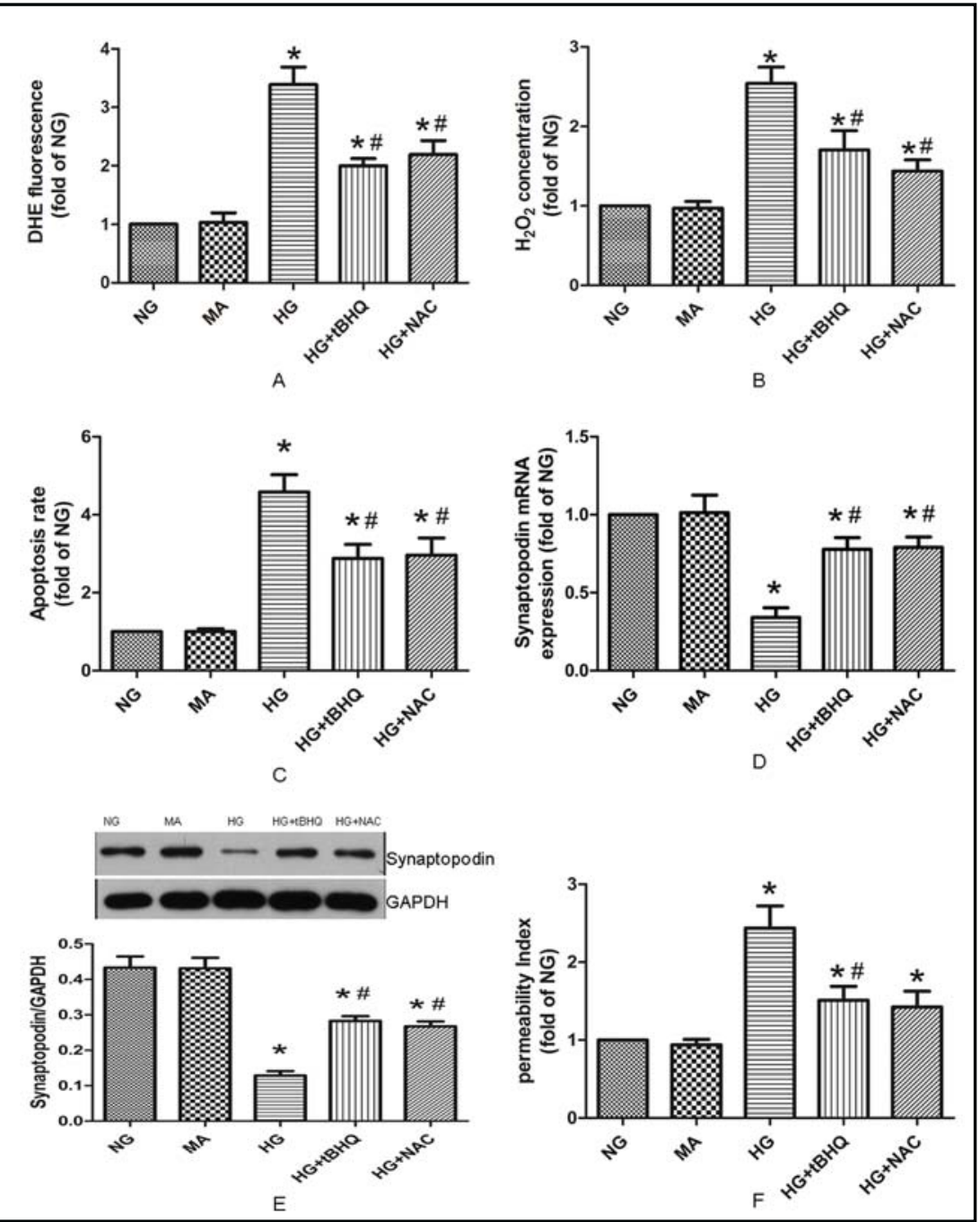

h. MA: Mouse podocytes incubated with mannitol(MA, 25mM) for $24 \mathrm{~h}$. HG: Mouse podocytes incubated high glucose $(25 \mathrm{mM})$ for $24 \mathrm{~h}$. HG+tBHQ: Mouse podocytes were pretreated with tert-Butylhydroquinone for $4 \mathrm{~h}$ and then incubated with high glucose $(25 \mathrm{mM})$ for $24 \mathrm{~h}$. HG+NAC: Mouse podocytes were pretreated with $\mathrm{N}$-acetylcysteine for $4 \mathrm{~h}$ and then incubated with high glucose $(25 \mathrm{mM})$ for $24 \mathrm{~h}$. Data are the mean \pm SEM, $* \mathrm{P}<0.05$ vs NG group, $\# \mathrm{P}<0.05$ vs $\mathrm{NG}$ group, $\mathrm{n}=3$.

rate of apoptosis, increased synaptopodin expression and lower BSA permeability in these podocytes compared with podocytes exposed to $H G(p<0.05)$ (Fig. 1).

$t B H Q$ protected mouse podocytes from excessive ROS and damages induced by $H G$

Pretreatment of $\mathrm{tBHQ}$ in podocytes incubated with $\mathrm{HG}$ can lower intracellular superoxide anion and hydrogen peroxide production $(p<0.05)$. However, these two parameters in these podocytes were higher than podocytes exposed to NG $(p<0.05)$. Simultaneously, pretreatment with $\mathrm{tBHQ}$ in these podocytes also lowered the extent of apoptosis, increased synaptopodin expression and lower BSA permeability in these podocytes compared with podocytes exposed to $H G(p<0.05)$ (Fig. 1).

tBHQ activated the Nrf2-ARE pathway in mouse podocytes incubated with HG

The activity of the Nrf2 antioxidant pathway in podocytes pretreated with tBHQ and then incubated with HG was higher than in podocytes incubated with HG $(p<0.05)$. Nrf2- 


\section{Cellular Physiology $\quad$ Cell Physiol Biochem 2014;34:891-902

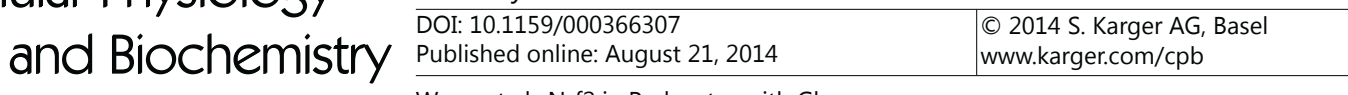

Fig. 2. Comparison of the expression of the Nrf2ARE pathway in podocytes exposed to different treatments. A: Comparison of Nrf2 transcriptional activity in podocyte from different groups. B: Comparison of Nrf-2, HO-1 and NQ01 mRNA expression in podocyte from different groups. C: Comparison of Nrf-2, HO-1 and NQ01 expression in podocyte from different groups. D: Comparison of nuclear Nrf-2 protein expression in podocyte from different groups. E: Comparison of cytoplasmic Nrf-2 protein expression in podocyte from different groups. NG: Mouse podocytes incubated with normal glucose (5 $\mathrm{mM}$ ) for $24 \mathrm{~h}$. MA:
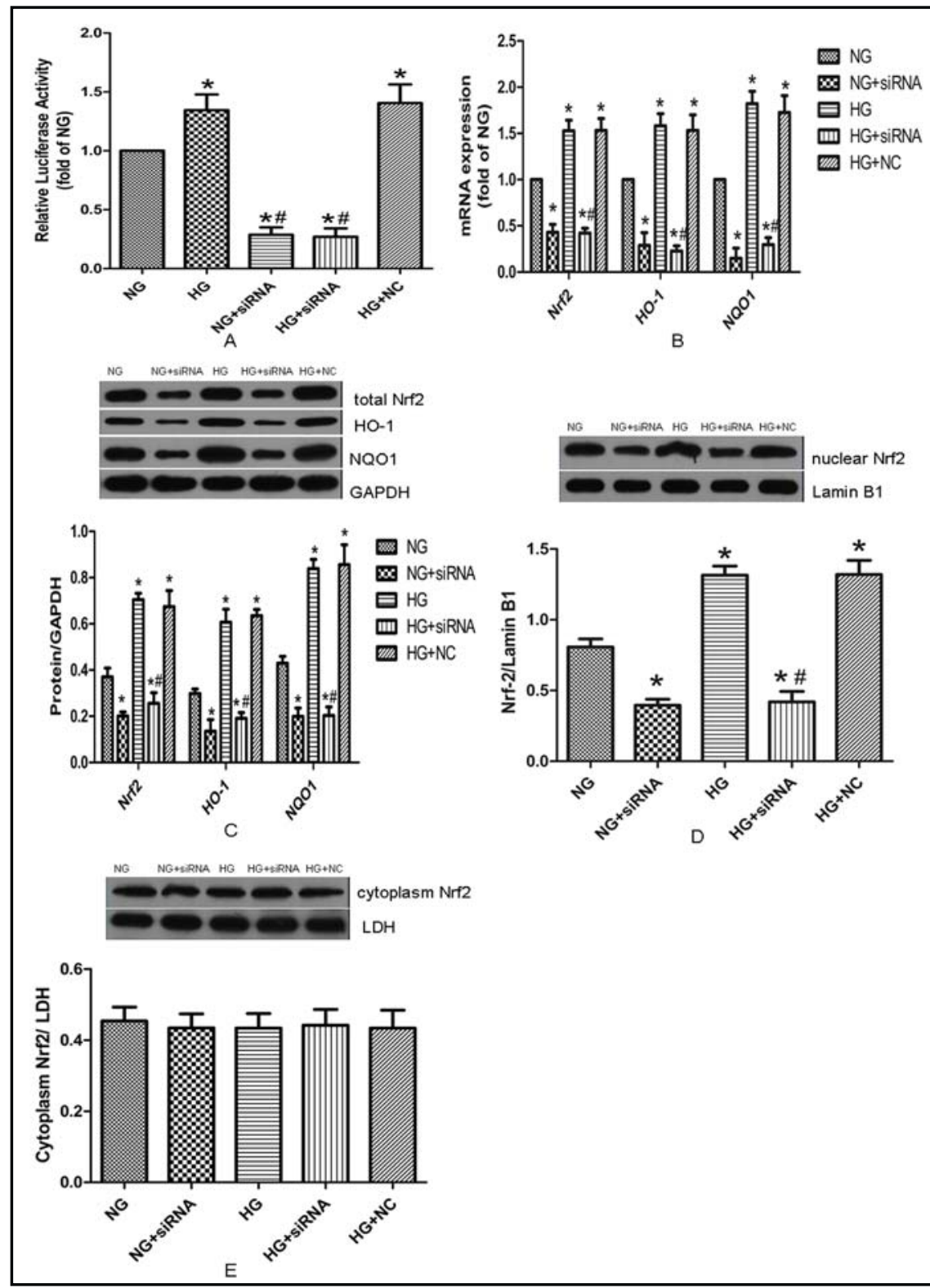

Mouse podocytes incubated with mannitol(MA, 25mM) for $24 \mathrm{~h}$. HG: Mouse podocytes incubated high glucose $(25 \mathrm{mM})$ for $24 \mathrm{~h}$. HG+tBHQ: Mouse podocytes were pretreated with tert-Butylhydroquinone for $4 \mathrm{~h}$ and then incubated with high glucose $(25 \mathrm{mM})$ for $24 \mathrm{~h}$. HG+NAC: Mouse podocytes were pretreated with $\mathrm{N}$-acetylcysteine for $4 \mathrm{~h}$ and then incubated with high glucose $(25 \mathrm{mM})$ for $24 \mathrm{~h}$. Data are the mean \pm SEM, * $\mathrm{P}<0.05$ vs $\mathrm{NG}$ group, $\# \mathrm{P}<0.05$ vs $\mathrm{NG}$ group, $\mathrm{n}=3$.

mRNA expression as well as enhanced total and nuclear Nrf2 protein expression in these podocytes pretreated with tBHQ were also higher than in podocytes incubated with HG $(p<0.05)$. Simultaneously, pretreatment with $\mathrm{tBHQ}$ in these podocytes increased expression of HO1 and NQO1 at protein and mRNA levels (p<0.05) (Fig. 2).

tBHQ decreased levels of MDA, but increased levels of SOD, GSH and CAT, in mouse podocytes incubated with $H G$

MDA levels in podocytes pretreated with $\mathrm{BBHQ}$ and then exposed to HG were lower than in podocytes incubated with HG $(\mathrm{p}<0.05)$. Levels of SOD, GSH and CAT in these podocytes were higher than in podocytes exposed to hyperglycemia ( $<<0.05$ ) (Fig. 3). 


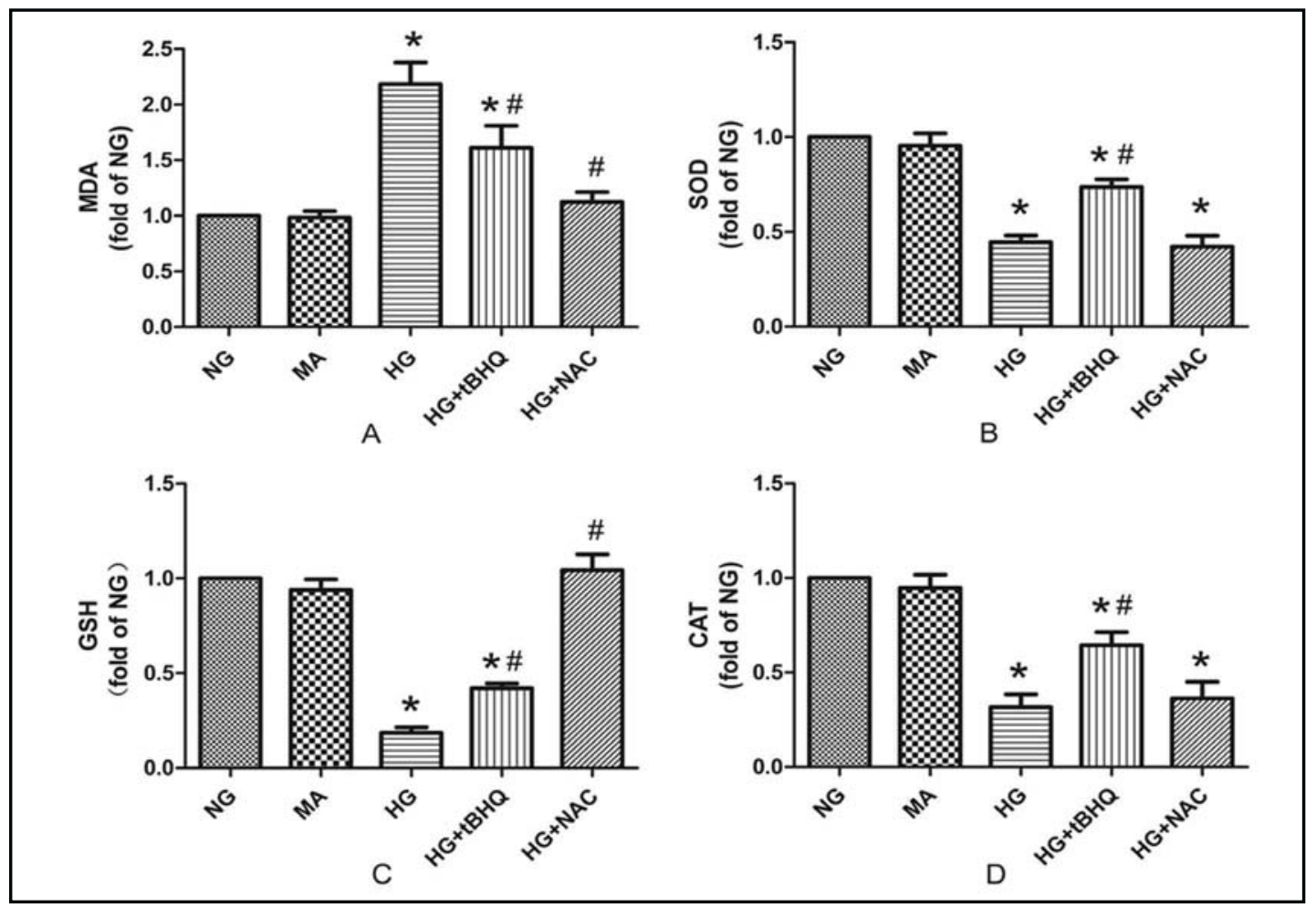

Fig. 3. Comparison of levels of malondialdehyde, superoxide dismutase, glutathione and catalase in podocytes exposed to different treatments. A: Comparison of levels of malondialdehyde in mouse podocytes mediated by high glucose $(25 \mathrm{mM})$. B: Comparison of levels of superoxide dismutase in mouse podocytes mediated by high glucose $(25 \mathrm{mM})$. C: Comparison of levels of glutathione in mouse podocytes mediated by high glucose $(25 \mathrm{mM})$. D: Comparison of levels of catalase in mouse podocytes mediated by high glucose (25 mM). NG: Mouse podocytes incubated with normal glucose (5 mM) for $24 \mathrm{~h}$. MA: Mouse podocytes incubated with mannitol(MA, 25mM) for $24 \mathrm{~h}$. HG: Mouse podocytes incubated high glucose (25 mM) for $24 \mathrm{~h}$. HG+tBHQ: Mouse podocytes were pretreated with tert-Butylhydroquinone for $4 \mathrm{~h}$ and then incubated with high glucose (25 mM) for $24 \mathrm{~h}$. HG+NAC: Mouse podocytes were pretreated with N-acetylcysteine for $4 \mathrm{~h}$ and then incubated with high glucose $(25 \mathrm{mM})$ for $24 \mathrm{~h}$. NG+siRNA: Nrf2 were knock-downed by siRNAs and then incubated with normal glucose $(5 \mathrm{mM})$ for $24 \mathrm{~h}$. HG+siRNA: Nrf2 were knock-downed by siRNAs and then incubated with high glucose $(25 \mathrm{mM})$ for $24 \mathrm{~h}$. $\mathrm{HG}+\mathrm{NC}$ : Mouse podocytes were transfected with the negative control of siRNA and then incubated with high glucose $(25 \mathrm{mM})$ for $24 \mathrm{~h}$. Data are the mean \pm SEM, *P $<0.05$ vs $\mathrm{NG}$ group, $\# \mathrm{P}<0.05$ vs $\mathrm{NG}$ group, $\mathrm{n}=3$.

Downregulation of Nrf2 by siRNA induced more excessive ROS and damages in mouse podocytes $H G$ than those podocytes exposed to $H G$

The level of intracellular superoxide anion and hydrogen peroxide production in podocytes that showed downregulated Nrf2 expression by siRNAs, then incubated with HG, was higher than in podocytes exposed to HG $(\mathrm{p}<0.05)$. Simultaneously, these podocytes had higher apoptosis rate, lower synaptopodin expression and higher BSA permeability in these podocytes compared with podocytes exposed to HG ( $p<0.05$ ) (Fig. 4).

Downregulation of Nrf2 by siRNAs inhibited the Nrf2-ARE pathway in mouse podocytes incubated with $H G$

The Nrf2-mRNA expression, total and nuclear Nrf2 protein expression, and Nfr2 transcriptional activity in podocytes that showed downregulated Nrf2 expression by siRNAs, then incubated with $\mathrm{HG}$ and $\mathrm{NG}$, were lower than in podocytes exposed to NG $(p<0.05)$. 


\section{Cellular Physiology Cell Physiol Biochem 2014;34:891-902

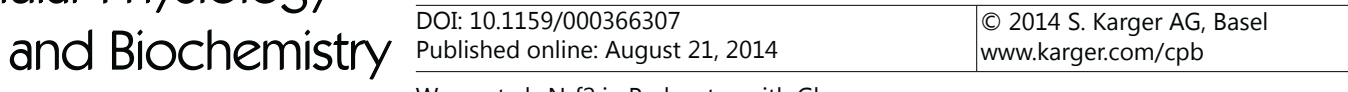

Fig. 4. The generation of ROS, apoptosis rate, synaptopodin expression and BSA permeability in podocytes down-regulated Nrf2 by siRNAs. A: The generation of superoxide anion generation in podocytes down-regulated Nrf2 by siRNAs. B: The generation of hydrogen peroxide production in podocytes downregulated Nrf2 by siRNAs. C: The apoptosis rate in podocytes down-regulated Nrf2 by siRNAs. D: The synaptopodin mRNA expression in podocytes down-regulated Nrf2 by siRNAs E: The synaptopodin expression in podocytes down-regulated Nrf2 by siRNAs. F: The BSA permeability in podocytes down-regulated Nrf2 by siRNAs. NG: Mouse podocytes incubated with normal glucose (5 $\mathrm{mM})$ for 24 h. NG+siRNA: Nrf2 were knock-downed by siRNAs and then these podocytes were incubated with nor-
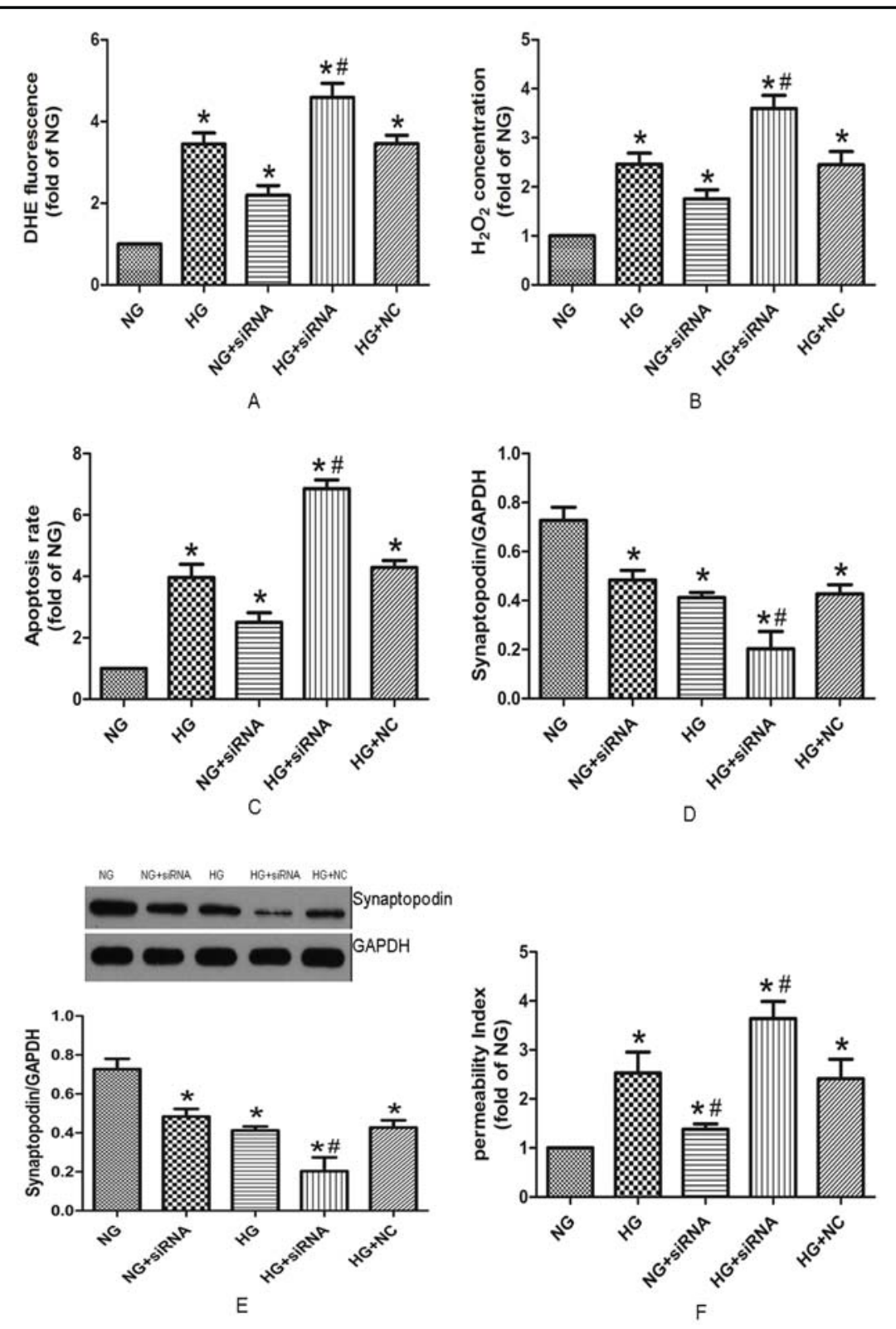

mal glucose $(5 \mathrm{mM})$ for $24 \mathrm{~h}$. HG: Mouse podocytes incubated high glucose (25 mM) for $24 \mathrm{~h}$. HG+siRNA: Nrf2 were knock-downed by siRNAs and then these podocytes were incubated with high glucose (25 mM) for $24 \mathrm{~h}$. HG+NC: Mouse podocytes were transfected with the negative control of siRNA and then incubated with high glucose $(25 \mathrm{mM})$ for $24 \mathrm{~h}$. Data are the mean $\pm \mathrm{SEM},{ }^{*} \mathrm{P}<0.05$ vs $\mathrm{NG}$ group, $\# \mathrm{P}<0.05$ vs $\mathrm{NG}$ group, $\mathrm{n}=3$.

Simultaneously, Nrf2 siRNAs also decreased expression of HO1 and NQO1 at protein and mRNA levels in these two podocyte regimens compared with podocytes incubated with NG $(p<0.05)$ (Fig. 5).

Downregulation of Nrf2 expression by siRNAs increased MDA levels to a greater extent, but decreased levels of SOD, GSH and CAT in mouse podocytes incubated with HG

MDA levels in podocytes that showed downregulated Nrf2 expression by siRNAs and were then incubated with HG were higher than in podocytes incubated with HG $(p<0.05)$. 

Cellular Physiology $\quad$ Cell Physiol Biochem 2014;34:891-902 and Biochemistry \begin{tabular}{l|l} 
DOI: 10.1159/000366307 \\
Publishea onine: August 21, 2014 & $\begin{array}{l}\text { O 2014 S. Karger AG, Basel } \\
\text { www.karger.com/cpb }\end{array}$
\end{tabular}
Wang et al.: Nrf2 in Podocytes with Glucose

Fig. 5. The expression of the Nrf2-ARE pathway in podocytes downregulated Nrf2 by siRNAs. A: Nrf2 transcriptional activity in podocytes d own - reg u lated Nrf2 by siRNAs. B: The Nrf-2, HO-1 and NQO1 mRNA expression in podocytes down-regulated Nrf2 by siRNAs. C: The Nrf-2, HO-1 and NQO1 expression in podocytes downregulated Nrf2 by siRNAs. D: The nuclear Nrf-2 protein expression in podocytes d own-regu lated Nrf2 by siRNAs. E: The cytoplasmic Nrf-2 protein expression in podocytes d own-regu lated $\mathrm{Nrf} 2$ by siRNAs. NG: Mouse podocytes incubated with normal

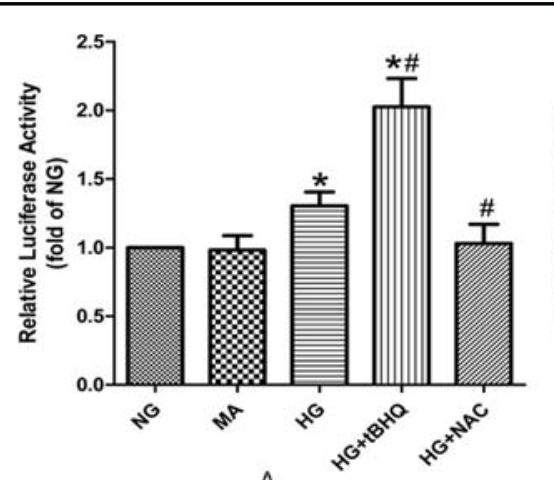

A
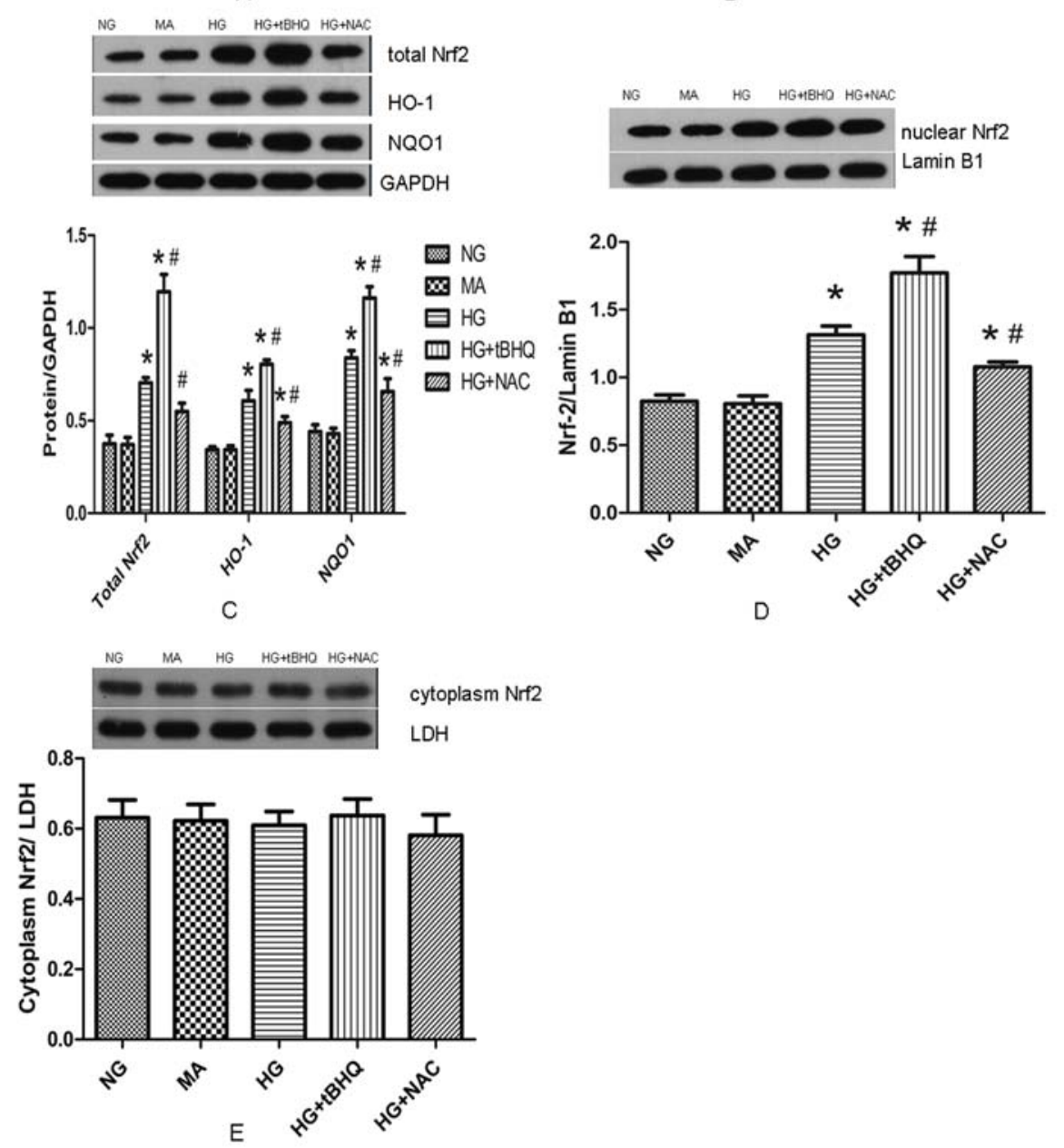

glucose ( $5 \mathrm{mM}$ ) for $24 \mathrm{~h}$. NG+siRNA: Nrf2 were knock-downed by siRNAs and then these podocytes were incubated with normal glucose ( $5 \mathrm{mM}$ ) for $24 \mathrm{~h}$. HG: Mouse podocytes incubated high glucose ( $25 \mathrm{mM})$ for 24 h. HG+siRNA: Nrf2 were knock-downed by siRNAs and then these podocytes were incubated with high glucose $(25 \mathrm{mM})$ for $24 \mathrm{~h}$. $\mathrm{HG}+\mathrm{NC}$ : Mouse podocytes were transfected with the negative control of siRNA and then incubated with high glucose $(25 \mathrm{mM})$ for $24 \mathrm{~h}$. Data are the mean $\pm \mathrm{SEM},{ }^{*} \mathrm{P}<0.05$ vs NG group, $\# \mathrm{P}<0.05$ vs NG group, $\mathrm{n}=3$.

Levels of SOD, GSH and CAT in these podocytes were lower than in podocytes exposed to HG $(p<0.05)$ (Fig. 6). 


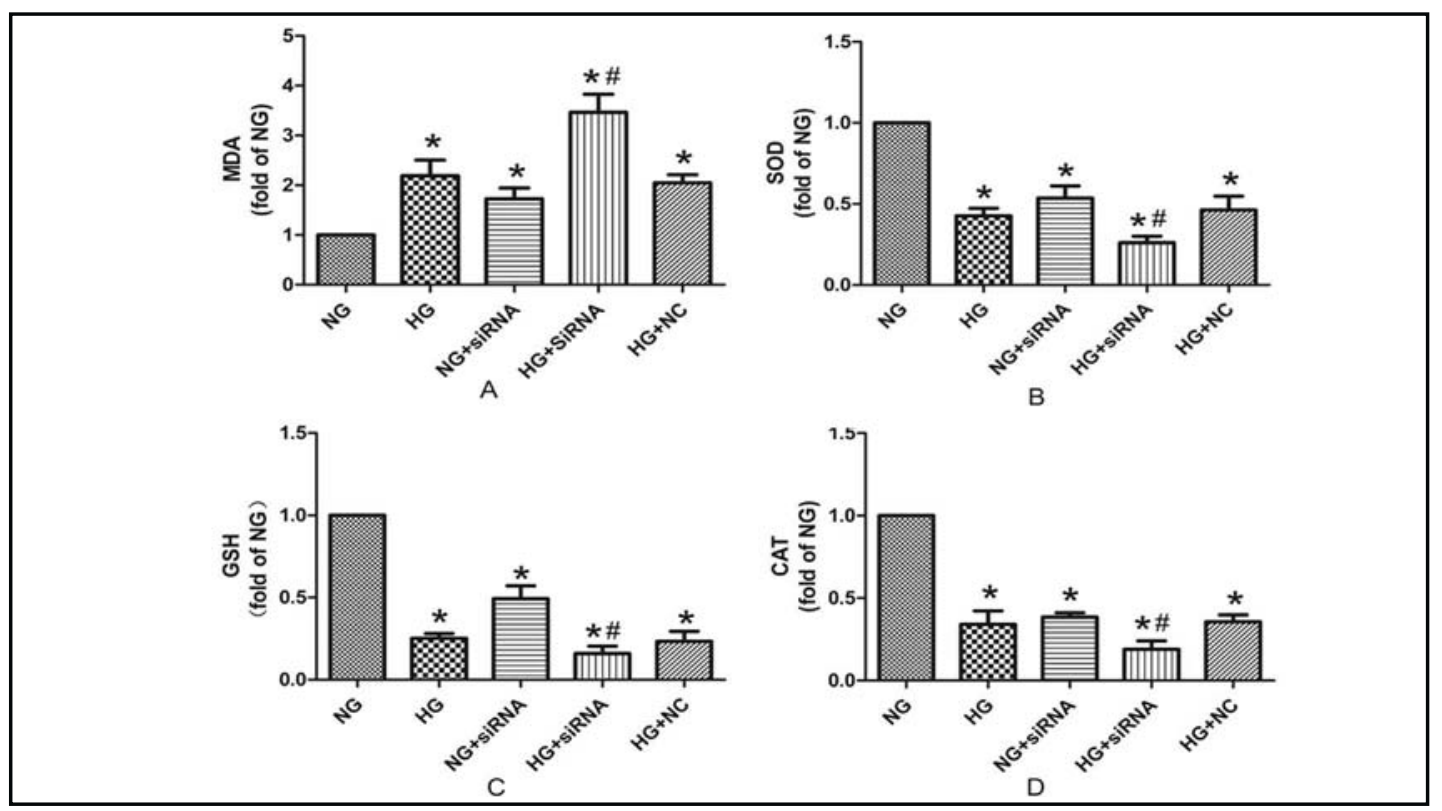

Fig. 6. The levels of malondialdehyde, superoxide dismutase, glutathione and catalase in podocytes downregulated Nrf2 by siRNAs. A: The levels of malondialdehyde in mouse podocytes down-regulated Nrf2 by siRNAs. B: The levels of superoxide dismutase in mouse podocytes down-regulated Nrf2 by siRNAs. C: The levels of glutathione in mouse podocytes down-regulated Nrf2 by siRNAs. D: The levels of catalase in mouse podocytes down-regulated Nrf2 by siRNAs. NG: Mouse podocytes incubated with normal glucose (5 $\mathrm{mM}$ ) for 24 h. NG+siRNA: Nrf2 were knock-downed by siRNAs and then these podocytes were incubated with normal glucose ( $5 \mathrm{mM}$ ) for $24 \mathrm{~h}$. HG: Mouse podocytes incubated high glucose (25 mM) for $24 \mathrm{~h}$. HG+siRNA: Nrf2 were knock-downed by siRNAs and then these podocytes were incubated with high glucose (25 mM) for 24 h. HG+NC: Mouse podocytes were transfected with the negative control of siRNA and then incubated with high glucose $\left(25 \mathrm{mM}\right.$ ) for $24 \mathrm{~h}$. Data are the mean $\pm \mathrm{SEM},{ }^{*} \mathrm{P}<0.05$ vs $\mathrm{NG}$ group, $\# \mathrm{P}<0.05$ vs NG group, $\mathrm{n}=3$.

\section{Discussion}

The present study demonstrated a protective effect of activation of the Nrf2-ARE pathway in podocytes mediated by hyperglycemia. Podocyte injuries induced by hyperglycemia were ameliorated by an activator of the Nrf2-ARE pathway tBHQ. When we downregulated Nrf2 expression by siRNAs, these podocytes showed more severe damage when exposed to HG.

We found that HG could induce the apoptosis of podocytes, inhibit synaptopodin expression, and increase BSA permeability. Simultaneously, ROS were increased in podocytes exposed to HG (i.e. intracellular superoxide anion and hydrogen peroxide production) as were cellular levels of MDA. We found that the defense enzymes levels of GSH, SOD and CAT in podocytes were decreased by HG. Taken together, these data suggested that hyperglycemia not only engendered excessive free radicals but also attenuated anti-oxidative factors [4], that's means the level of ROS exceeds the defense mechanisms, the pododyte is said to be in a state of oxidative stress [21]. To identify the role of ROS in podocyte injury, the classical antioxidant reagent $\mathrm{N}$-acetylcysteine, which reacted with superoxide, hydrogen-peroxide, peroxynitrite and served as a precursor of cysteine for GSH synthesis [22], was used to pretreat these podocytes. We found that NAC could attenuate the podocyte damage induced by HG, which further confirmed the role of ROS in the podocyte damage induced by HG.

Nrf2 regulates the transcription of a plethora of cytoprotective genes, including antioxidant and phase-II detoxifying enzymes. In this context, Nrf2 has emerged as a central regulator of endogenous antioxidant pathways. Transgenic studies have established that the kidneys from $\mathrm{Nrf2}^{-{ }^{--}}$animals are more susceptible to streptozotocin-induced DM, whereas compounds that increase Nrf2 activity (e.g., antroquinonol, tBHQ, and triterpenoids) may 


\section{Cellular Physiology and Biochemistry}

Cell Physiol Biochem 2014;34:891-902

\begin{tabular}{l|l}
\hline DOI: $10.1159 / 000366307$ & C 2014 S. Karger AG, Basel
\end{tabular}

www.karger.com/cpb

Wang et al.: Nrf2 in Podocytes with Glucose

be renoprotective $[23,24]$. Protection against activation of the Nrf2 pathway has been documented in different cells, especially endothelial cells [13-16]. However, data on the role of the Nrf2-ARE pathway in podocytes incubated with HG are lacking. This is the first study to show that activation of the Nrf2-ARE pathway can attenuate the podocyte injury induced by hyperglycemia. Under oxidative stress, Nrf2 is translocated into the nucleus and activates the ARE pathway [25], and the glomeruli of subjects with DKD have elevated Nrf2 levels. It is not surprising to find that Nrf2 mRNA expression, Nrf2 nuclear protein levels, and target protein expression were increased in podocytes exposed to HG. However, it is not sufficient to overcome the deleterious effects of hyperglycemia on podocytes because anti-oxidative factors such as SOD, GSH and CAT were inhibited under these conditions. Hence, we used tBHQ to activate the Nrf2-ARE pathway. This was documented by the enhancement of Nrf2 mRNA expression and nuclear Nrf2 proteins, activity of the Nrf2 antioxidant pathway, target protein expression (e.g., NQO1 and HO1) and anti-oxidative factors such as SOD, GSH and CAT to lower oxidative stress and alleviate the podocyte damage induced by hyperglycemia. Conversely, podocytes exposed to HG showed more severe injury and higher ROS production when we downregulated Nrf2 expression by siRNAs. All of these results supported the notion that activation of the Nrf2-ARE pathway could alleviate the podocyte injury mediated by HG.

However, there are some limitations should be considered when assessed the paper. Firstly, mouse podocytes cannot replace human podocytes. Secondly, it is just a vitro study, vivo study is needed to further confirm the result.

Collectively, these results showed that protection against activation of the Nrf2-ARE pathway in podocytes exposed to hyperglycemia. Hence, regulation of the Nrf2-ARE pathway by pharmacological or molecular means could serve as a potential adjunct therapy to combat oxidative stress and inhibit the development of DKD.

\section{Disclosure Statement}

We declared no conflicts of interest, financial or otherwise.

\section{Reference}

1 Giacco F, Brownlee M: Oxidative stress and diabetic complications. Cirs Res 2010;107:1058-1070.

2 Brownlee M: The pathobiology of diabetic complications: a unifying mechanism. Diabetes 2005;54:16151625.

3 West IC: Radicals and oxidative stress in diabetes. Diabet Med 2000;17:171-180.

-4 Nishikawa T, Edelstein D, Du XL, Yamagishi S, Matsumura T, Kaneda Y, Yorek MA, Beebe D, Oates PJ, Hammes HP, Giardino I, Brownlee M: Normalizing mitochondrial superoxide productionblocks three pathways of hyperglycaemic damage. Nature 2000;404:787-790.

-5 Jim B, Ghanta M, Qipo A, Fan Y, Chuang PY, Cohen HW, Abadi M, Thomas DB, He JC: Dysregulated nephrin in diabetic nephropathy of type 2 diabetes: a cross sectional study. PLoS One Doi: 10.1371/journal. pone.0036041.

6 Shankland SJ, Pippin JW, Reiser J, Mundel P: Podocytes in culture: past, present, and future. Kidney Int 2007;72:26-36.

7 Hoshi S, Shu Y, Yoshida F, Inagaki T, Sonoda J, Watanabe T, Nomoto K, Nagata M: Podocyte injury promotes progressive nephropathy in zucker diabetic fatty rats. Lab Invest 2002;82:25-35.

-8 Coimbra TM, Janssen U, Gröne HJ, Ostendorf T, Kunter U, Schmidt H, Brabant G, Floege J: Early events leading to renal injury in obese Zucker rats with type II diabetes. Kidney Int 2000;57:167-182.

9 Menini S, Iacobini C, Oddi G, Ricci C, Simonelli P, Fallucca S, Grattarola M, Pugliese F, Pesce C, Pugliese G: Increased glomerular cell (podocyte) apoptosis in rats with streptozotocin-induced diabetes mellitus: role in the development of diabetic glomerular disease. Diabetologia 2007;50:2591-2599.

10 Biswal S, Thimmulappa RK, Harvet CJ: Experimental therapeutias of nrf2 as a target for prevention of bacterial exacerbations in COPD. Proc Am Thorac Soc 2012;9:47-51. 


\section{Cellular Physiology and Biochemistry}

Cell Physiol Biochem 2014;34:891-902

\begin{tabular}{l|l}
\hline DOI: $10.1159 / 000366307$ & (C) 2014 S. Karger AG, Basel
\end{tabular}

Wang et al.: Nrf2 in Podocytes with Glucose

11 Jaiswal AK: Regulation of genes of encoding NADPH:quinine oxidoreductases. Free Radic Biol Med 2000;29:254-262.

12 Harvey CJ, Thimmulappa RK, Singh A, Blake DJ, Ling G, Wakabayashi N, Fujii J, Myers A, Biswal S: Nrf2 regulated glutathione recucling independent of biosyntheisi is critical for cell survival during oxidative stress. Free Radic Biol Med 2009;46:443-453.

13 Zhong Q, Mishra M, Kowluru RA: Transcription factor Nrf2-mediated antioxidant defense system in the development of diabetic retinopathy. Invest Ophthalmol Vis Sci 2013;54:3941-3941.

-14 Tsai CY, Wang CC, Lai TY, Tsu HN, Wang CH, Liang HY, Kuo WW: Antioxidant effect of diallyl trisulfide on high glucose-indueed apoptosis are mediated by the PI3K-Akt-dependent activation of Nrf2 in cardiomyocytes. Int J Cardiol 2013;30:1286-1297.

15 Li H, Wang F, Zhang L, Cao Y, Liu W, Hao J, Liu Q, Duan H: Modulation of Nrf2 expression alters high glucose-induced oxidative stress and antioxidant gene expression in mouse mesangial cells. Cell Signal 2011;23:1625-1632.

-16 Ungvari Z, Bailey-Downs L, Gautam T, Jimenez R, Losonczy G, Zhang C, Ballabh P, Recchia FA, Wilkerson DC, Sonntag WE, Pearson K, de Cabo R, Csiszar A: Adaptive induction of NF-E2-related factor-2 driven antioxidant genes in endothelial cells in respone to hyperglycemia. Am J Physiol Heart Circ Physiol 2011;300:H1133-1140.

17 Wang C, Liu X, Tang Y, Peng H, Ye Z, Zhang J, Tang H, Lou T: Medium from meangial cells incubated with aggregated IgA1 from IgA nephropathy patients reduces podocyte adhesion through activation of the rennin angiotensin system. Swiss Med Wkly Doi: 10.4414/smw.2011.13304 .

18 Wang C, Liu X, Ke Z, Tang Y, Li CC, Li CM, Ye Z, Zhang J, Lou T: Mesangial medium form IgA nephropathy patients induces podocyte epithelial to mesenchymal transition through activation of the phosphatidyl inositol-3-kinase/Akt signaling pathway. Cell Physiol Biochem 2012;29:743-752.

$\checkmark 19$ Jirapan Moungjaroen, Ubonthip Nimmannit, Patrick S: Callery:Reactive Oxygen Species Mediate Caspase Activation and Apoptosis Induced by Lipoic Acid in Human Lung Epithelial Cancer Cells through Bcl-2 Down-Regulation. J Pharmacol Exp Ther 2006;319:1062-1069.

20 Kumar S, Allen DA, Kieswich JE, Patel NS, Harwood S, Mazzon E, Cuzzocrea S, Raftery MJ, Thiemermann C, Yaqoob MM: Dexamethasone Ameliorates Renal Ischemia-Reperfusion Injury. J Am Soc Nephrol 2009;20:2412-2425.

-21 Forbes JM, Coughlan MT, Cooper ME: Oxidative stress as a major culprit in kidney disease in diabetes. Diabetes 2008;57:1446-1454.

22 Samuni Y, Goldstein S, Dean OM, Berk M: The chemistry and biological activities of N-acetylcysteine. Biochim Biophys Acta. 2013;1830:4117-4129.

23 Jiang T, Huang Z, Lin Y, Zhang Z, Fang D, Zhang DD:The protective role of nrf2 in streptozotocin-induced diabetic nephropathy. Diabetes 2010;59:850-860.

24 Zheng H, Whitman SA, Wu W, Wondrak GT, Wong PK, Fang D, Zhang DD: Therapeutic potential of Nrf2 activators in streptozotocin-induced diabetic nephropathy. Diabetes 2011;60:3055-3066.

-25 Piantadosi CA, Carraway MS, Babiker A, Suliman HB: Heme oxygenase-1 regulates cardiac mitochondrial biogenesis via nrf2-mediated transcriptional control of nuclear respiratory factor. Cir Res 2008;103:12321240. 